\title{
Editorial: Design and Applications of Metal- and Metal Oxide-Based Antibacterial Materials
}

\author{
Gopalu Karunakaran ${ }^{1 *}$, Eun-Bum Cho ${ }^{1 *}$, Denis Kuznetsov ${ }^{2}$ and Govindan Suresh Kumar ${ }^{3}$ \\ ${ }^{1}$ Department of Fine Chemistry, Seoul National University of Science and Technology, Seoul, South Korea, ${ }^{2}$ Department of \\ Functional Nanosystems and High-Temperature Materials, National University of Science and Technology "MISiS," Leninskiy Pr. \\ 4, Moscow, Russia, ${ }^{3}$ Department of Physics, K. S. Rangasamy College of Arts and Science (Autonomous), Tiruchengode, India
}

Keywords: metal nano particle, metal oxide nanoparticles, antibacterial properties, biomaterials, nontoxic materials

\section{Editorial on the Research Topic}

\section{Design and Applications of Metal- and Metal Oxide-Based Antibacterial Materials}

The occurrence of harmful bacteria leads to a life-threatening to human through different ways of transmission (Sanchez and Doron, 2017). The bacteria can cause major infections such as tuberculosis, strep throat, cholera, food poisoning, staph infections and pneumonia (Sanchez and Doron, 2017). Bacteria like B. anthracis identified as "Anthrax" was also used as a bioweapon during the world war (Goel, 2015). A case of anthrax was found in the United States during 2001, in which anthrax-containing envelopes were sent by mail, affecting 22 persons, and it was classified as bioterrorism (Goel, 2015). Currently, serious illness due to the antibiotic-resistant strains also leads to the complications and negative effectiveness against the cure of bacterial infections. Further, the bacterial contamination is also observed in food products, which leads to serious impact in the industries of dairy, food, and marine and so on (Chatterjee and Abraham, 2018). Moreover, the bacterial infections are not only limited to the food industry, but also pose a serious threat to the leather industry and water management systems. The occurrence of biofilms containing bacteria in the leather products and water leads to the major health issues (Skóra et al., 2014). Thus, it is very essential for the development of materials which can resist the growth and development of these kinds of harmful bacteria.

Based on recent research and development in various scientific and technological fields, new therapeutic molecules, drugs, materials and coating technologies are being developed to control various bacterial infections and challenges. Among various fields, nanotechnology is currently booming for the development of novel materials for various application fields such as electronics, biomaterials, and environment. Nanotechnology includes the synthesis of nanoparticles by different methods for various applications (Bayda et al., 2020). Any materials which is at least in the range of $<100 \mathrm{~nm}$ in the diameter is considered as nanoparticles (Bayda et al., 2020). Generally, nanoparticles are present in the nature in the form of clays, ores, minerals and bacteria. It has been used by potters and glassmakers since ancient times, and especially Roman Lycurgus cups were later found to contain copper, silver and gold nanoparticles (Cheng, 2014). Mostly nanoparticles can be classified depending on the morphology, size, chemical and physical properties. The major group of nanoparticles are based on ceramic nanoparticles, polymeric nanoparticles, carbon based nanoparticles, semiconductor nanoparticles, lipid based nanoparticles, metal and metal oxide based nanoparticles (Khan et al., 2019).

Among the various types of nanoparticles available, metal and metal oxide are superior in terms of low toxicity and ease of synthesis (Khan et al., 2019). Metal oxide-based materials possess exceptional antibacterial properties by inhibiting the metabolic pathways of bacteria, even multi-resistant bacteria. Multi-resistant bacteria are bacteria that are resistant to the majority of available 
antibiotics for treatment. Metal oxides have site-specific properties similar to antibiotics which recognize metalloproteins and metal transport chains (McNamara and Tofail, 2017). Hence, these tremendous properties make metal oxide-based materials excellent antibacterial materials.

Metallic nanomaterials like copper, gold, silver, titanium and zinc are found to be a perfect antimicrobial agent (Nikolova and Chavali, 2020). The smaller size of metallic nanomaterial gives higher surface area which provides a strong affinity toward the surface attachments in different bacteria and bacterial biofilms, which results in the bactericidal effects. The main pathways by which metal nanoparticles exert a bactericidal effect are the generation of reactive oxygen species (ROS), binding to cytoplasmic proteins to inhibit their function, DNA interactions and cell wall lysis (Dizaj et al., 2014). It is known that metal nanomaterials with excellent antibacterial activity are more promising and non-toxic against harmful microorganisms than currently available antibiotics. Therefore, the use of metal nanomaterials and the development of synthetic methods are increasing day by day. Thus, it is very necessary to understand the current situation on the advancement of metal nanomaterial synthesis and applications.

With this in mind, this Research Topic aims to bring together recent advances in the development of metal- and metal oxidebased antibacterial materials via reliable, ecofriendly, and costeffective approaches to control multi-drug resistant bacteria. Thus, we welcome contributions that focus on the development, design, and application of antibacterial materials in the form of Review or Original Research articles. Key themes include, but are not limited to, the following:

- Design and characterization of novel metal- and metal oxidebased antibacterial materials, including nanoparticles and coatings

- Green synthesis or ecofriendly synthesis methods for metaland metal oxide-based antibacterial materials

- New antibacterial metal or metal oxide composites

As an outcome, various research papers and review papers have been submitted. The collection of original research articles and review papers includes current research topics based on innovative concepts and ideas for the design and application of metal- and metal oxide-based antibacterial materials. Papers have been published after rigorous peer review by experts in their specific field of study for timely submission of manuscripts by researchers. Jan et al. showed an excellent biogenic synthesis

\section{REFERENCES}

Bayda, S., Adeel, M., Tuccinardi, T., Cordani, M., and Rizzolio, F. (2020). The History of Nanoscience and Nanotechnology: From Chemical-Physical Applications to Nanomedicine. Molecules 25, 112. doi:10.3390/ molecules 25010112

Chatterjee, A., and Abraham, J. (2018). Microbial Contamination, Prevention, and Early Detection in Food Industry, Microb. Contam. Food Degrad., 21-47. doi:10.1016/b978-0-12-811515-2.00002-0 of zinc oxide $(\mathrm{ZnO})$ nanoparticles by using Himalayan columbine extracts. The produced $\mathrm{ZnO}$ nanoparticles were excellent antimicrobial and anti-parasitic. Garza-Cervantes et al. demonstrated an efficient method of treating bacterial infections with metallic micronutrients and antibiotic combinations as a therapeutic agent. In this study, $\mathrm{Zn}^{2+}$, $\mathrm{Cu}^{2+}, \mathrm{Cd}^{2+}, \mathrm{Ni}^{2+}$ and $\mathrm{Co}^{2+}$ were found to enhance the susceptibility to Staphylococcus aureus and Escherichia coli when used with kanamycin and ampicillin. Qi et al. described the detailed mechanisms of action of cerium oxide [Ce(III) and $\mathrm{Ce}(\mathrm{IV})]$ nanoparticles. This work also describes the synthesis and antibacterial actions of cerium oxide nanoparticles. The mechanism of cerium oxide nanoparticles involved in antibacterial actions is due to electrostatic interaction, membrane blockage, cell membrane damage, oxidative stress, and DNA oligomer hydrolysis.

Abudula et al. showed that the metal or metal oxide nanoparticles incorporated into $3 \mathrm{D}$ printed thermoplastics can be used effectively in daily life and in medical devices. The metal and metal oxide nanoparticles' incorporation leads to the prevention of bacterial contamination by various bactericidal mechanisms. Metal and metal oxide also did not affect the function and the integrity of the thermoplastic. We, as the guest editors of this research topic, would like to thank all the researchers for their valuable contribution to this research topic. We would also like to thank all the reviewers, all the supporting and technical staff at Frontiers Editorial, for their invaluable support throughout the successful completion of this research topic.

\section{AUTHOR CONTRIBUTIONS}

All authors listed have made a substantial, direct, and intellectual contribution to the work and approved it for publication.

\section{FUNDING}

E-BC and GK were supported by Brain Pool Program through the National Research Foundation of Korea (NRF) funded by the Ministry of Science and ICT (Grant no. 2018H1D3A1A01037054). GK also acknowledges supports under the Basic Science Research Program through the National Research Foundation of Korea funded by the Ministry of Education (NRF2019R1I1A1A01062458).

Cheng, X. (2014). Nanostructures: Fabrication and Applications. Nanolithography Art Fabr. Sawston, United Kingdom, 348-375. doi:10.1533/9780857098757.348 Nanoelectron. Nanophotonic Devices Syst

Dizaj, S. M., Lotfipour, F., Barzegar-Jalali, M., Zarrintan, M. H., and Adibkia, K. (2014). Antimicrobial Activity of the Metals and Metal Oxide Nanoparticles. Mater. Sci. Eng. C 44, 278-284. doi:10.1016/j.msec.2014.08.031

Goel, A. K. (2015). Anthrax: A Disease of Biowarfare and Public Health Importance. Wjcc 3, 20. doi:10.12998/wjcc.v3.i1.20

Khan, I., Saeed, K., and Khan, I. (2019). Nanoparticles: Properties, Applications and Toxicities. Arabian J. Chem. 12, 908-931. doi:10.1016/j.arabjc.2017.05.011 
McNamara, K., and Tofail, S. A. M. (2017). Nanoparticles in Biomedical Applications. Adv. Phys. X 2, 54-88. doi:10.1080/23746149.2016.1254570

Nikolova, M. P., and Chavali, M. S. (2020). Metal Oxide Nanoparticles as Biomedical Materials. Biomimetics 5, 27. doi:10.3390/ BIOMIMETICS5020027

Sanchez, E., and Doron, S. (2017). Bacterial Infections: Overview. Cambridge, MA: Int. Encycl. Public Heal., 196-205. doi:10.1016/B978-0-12-803678-5.00030-8

Skóra, J., Gutarowska, B., Stępień, Ł., Otlewska, A., and Pielech-Przybylska, K. (2014). The Evaluation of Microbial Contamination in the Working Environment of Tanneries. Med. Pr. 65, 15-32. doi:10.13075/ mp.5893.2014.005
Conflict of Interest: The authors declare that the research was conducted in the absence of any commercial or financial relationships that could be construed as a potential conflict of interest.

Copyright (๑) 2021 Karunakaran, Cho, Kuznetsov and Suresh Kumar. This is an open-access article distributed under the terms of the Creative Commons Attribution License (CC BY). The use, distribution or reproduction in other forums is permitted, provided the original author(s) and the copyright owner(s) are credited and that the original publication in this journal is cited, in accordance with accepted academic practice. No use, distribution or reproduction is permitted which does not comply with these terms. 